\title{
Electriflow: Soft Electrohydraulic Building Blocks for Prototyping Shape-changing Interfaces
}

\author{
Purnendu \\ ATLAS Institute, University of \\ Colorado Boulder \\ Boulder, CO, USA \\ purnendu@colorado.edu
}

\author{
Christoph Keplinger \\ Department of Mechanical \\ Engineering, University of Colorado \\ Boulder \\ Boulder, CO, USA \\ christoph.keplinger@colorado.edu
}

\author{
Sasha M Novack \\ ATLAS Institute, University of \\ Colorado Boulder \\ Boulder, CO, USA \\ sasha.novack@colorado.edu
}

\author{
Mirela Alistar \\ ATLAS Institute, University of \\ Colorado Boulder \\ Boulder, CO, USA \\ mirela.alistar@colorado.edu
}

\author{
Eric Acome \\ Department of Mechanical \\ Engineering, University of Colorado \\ Boulder \\ Boulder, CO, USA \\ eric.acome@colorado.edu \\ Mark D Gross \\ ATLAS Institute, University of \\ Colorado Boulder \\ Boulder, CO, USA \\ mdgross@colorado.edu
}

\section{Carson Bruns \\ ATLAS Institute, University of \\ Colorado Boulder \\ Boulder, CO, USA \\ carson.bruns@colorado.edu}

\author{
Daniel Leithinger \\ ATLAS Institute, University of \\ Colorado Boulder \\ Boulder, CO, USA \\ daniel.leithinger@colorado.edu
}
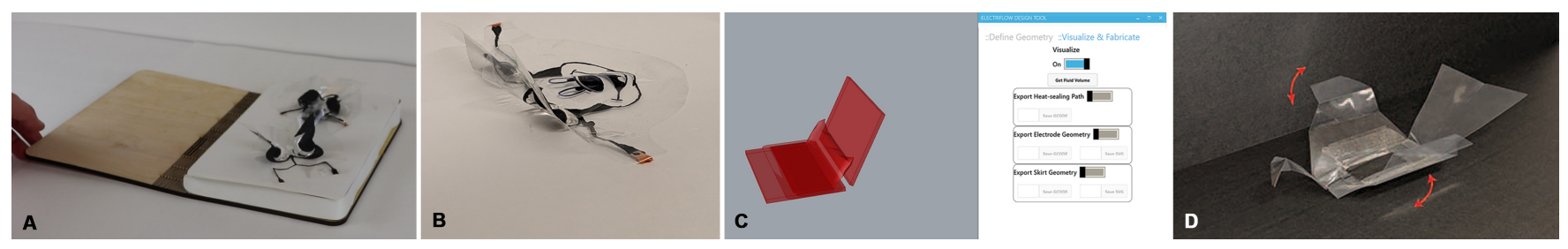

Figure 1: Electrohydraulic actuators offer a silent, rapid, compact and low-cost alternative for developing shape-changing interfaces. Here, we show example application prototypes as (A) Tangible animation integrated in a book, (B) physical character animation and (C) a software tool to facilitate prototyping, (D) application prototype for actuating origami creases.

\footnotetext{
ABSTRACT

We present Electriflow: a new class of soft electrohydraulic actuators as building blocks for prototyping shape-changing interfaces. These actuators are silent and fast in operation and can be fabricated with commodity materials. Electriflow generates an immediate hydraulic force upon electrostatic activation without an external fluid supply source, enabling a simple and compact self-contained design. This paper describes the materials and mechanisms of these shape-changing building blocks, as well as the underlying fabrication process, which includes a software tool that assists in their

Permission to make digital or hard copies of all or part of this work for personal or classroom use is granted without fee provided that copies are not made or distributed for profit or commercial advantage and that copies bear this notice and the full citation on the first page. Copyrights for components of this work owned by others than the author(s) must be honored. Abstracting with credit is permitted. To copy otherwise, or republish, to post on servers or to redistribute to lists, requires prior specific permission and/or a fee. Request permissions from permissions@acm.org.

DIS '21, June 28-fuly 2, 2021, Virtual Event, USA

(c) 2021 Copyright held by the owner/author(s). Publication rights licensed to ACM. ACM ISBN 978-1-4503-8476-6/21/06 ..\$15.00

https://doi.org/10.1145/3461778.3462093
}

design, shape visualization and construction. Finally, we explore four classes of application prototypes: tangible animation, actuating origami creases, shape-changing phone, and shape-changing bowl.

\section{CCS CONCEPTS}

- Human-centered computing $\rightarrow$ User interface toolkits; $\mathbf{H u}$ man computer interaction ( $\mathrm{HCI}$ ).

\section{KEYWORDS}

electrohydraulic actuation; shape-changing interfaces; tangible animation; fabrication.

\section{ACM Reference Format:}

Purnendu, Sasha M Novack, Eric Acome, Christoph Keplinger, Mirela Alistar, Mark D Gross, Carson Bruns, and Daniel Leithinger. 2021. Electriflow: Soft Electrohydraulic Building Blocks for Prototyping Shape-changing Interfaces. In Designing Interactive Systems Conference 2021 (DIS '21), fune 28-fuly 2, 2021, Virtual Event, USA. ACM, New York, NY, USA, 11 pages. https: //doi.org/10.1145/3461778.3462093 


\section{INTRODUCTION}

Electrohydraulic mechanisms are ubiquitous in the biological world. Beyond the complex chemical and biological function of cells [18], and vital organs (like human heart and lungs) [24], nature prefers electrohydraulic phenomena to drive shape change [28]. The compactness, immediate actuation and silent operation of these mechanisms enrich the ability of living organisms to convey information or perform desired actions. For example, a pufferfish threatens a predator by filling its elastic stomach with fluid (air or water) under electrohydraulic effects [8]. Plants like Venus Flytrap catch their prey through leaf closure employing electrohydraulic forces [11, 43]. The drastic shape transformation in certain species of octopus is also a result of electrohydraulic phenomena [3, 4]. Electrohydraulic processes drive shape change in plants and animals, and augment their ability to communicate and interact.

Communication and interaction through shape change is an emerging area of research in the field of Human-Computer Interaction (HCI). Such interactive systems, known as shape-changing interfaces, physically transform their shape to render digital information and provide dynamic affordances depending on the user and context [33]. Though bio-inspired systems for prototyping shapechanging interfaces is an active area of research [13, 44, 45], the current techniques suffer from limitations like being slow, noisy and bulky [2]. A better prototyping technology for such interfaces would enable immediate actuation, noiseless operation and compact integration akin to electrohydraulic shape-changing mechanisms found in nature. In this work, we present Electriflow: soft electrohydraulic actuators as building blocks for prototyping interactive systems through shape-change. Electriflow offers numerous advantages as a silent, fast, soft, and self-contained technology.

Despite previous attempts to build soft electrohydraulic actuators $[1,19]$, to the best of our knowledge, such building blocks are yet to be developed for shape-changing interfaces. Electriflow building blocks are novel bendable actuators capable of sharp hingelike and smooth-curve type folds when activated by electric fields. We present three novel actuator geometries which operate by the interplay of the electrostatic and hydraulic forces. Although a DC electric voltage drives the activation of Electriflow, it is the redistribution of the working fluid that causes a shape change. We envision these electrohydraulic building blocks can be leveraged by designers and other Human-Computer Interaction (HCI) researchers who require high actuation speeds, noiseless operation and compact integration for prototyping shape-changing interfaces. The core contributions of this work are as follows:

- Three novel actuator geometries-hinge-bending, crescent, quarter-moon-as building blocks for prototyping shapechanging interfaces, capable of producing sharp-hinge and smooth-curve bends.

- A software tool to aid the design, visualization and fabrication of shape-changing interfaces prototyped through Electriflow.

- Application prototypes that demonstrate how Electriflow enriches the interaction design possibilities for shape-changing interfaces, including tangible animation, actuating origami creases, shape-changing phone, and shape-changing bowl.

\section{RELATED WORK}

Our work builds on previous developments in bioinspired shapechanging interfaces and electrohydraulic actuators. We discuss both these types, critically looking at the current field with respect to the speed of actuation, portability and noise of operation.

\subsection{Bioinspired Shape-Changing Interfaces}

Bioinspired Shape-Changing Interfaces take inspiration from natural world by employing soft actuation elements, which are driven by developments in soft robotics and materials science [32]. For example, bioLogic integrates hygromorphic Natto cells into biohybrid shape-changing materials such as sweat-responsive garments or steam-triggered tea leaves [45]. Transformative appetite employs hydrogels derieved from seaweed to produce edible and shapechanging materials [41]. Printed Paper Actuator [40], Thermorph [5] and UniMorph [16] use heat to activate shape change in composite materials. These actuators generate mechanical force by coupling two materials with differential expansion rates under various stimuli. Despite being compact and silent in operation, their actuation modes are limited by slow response times.

Denotes Supported Attributes

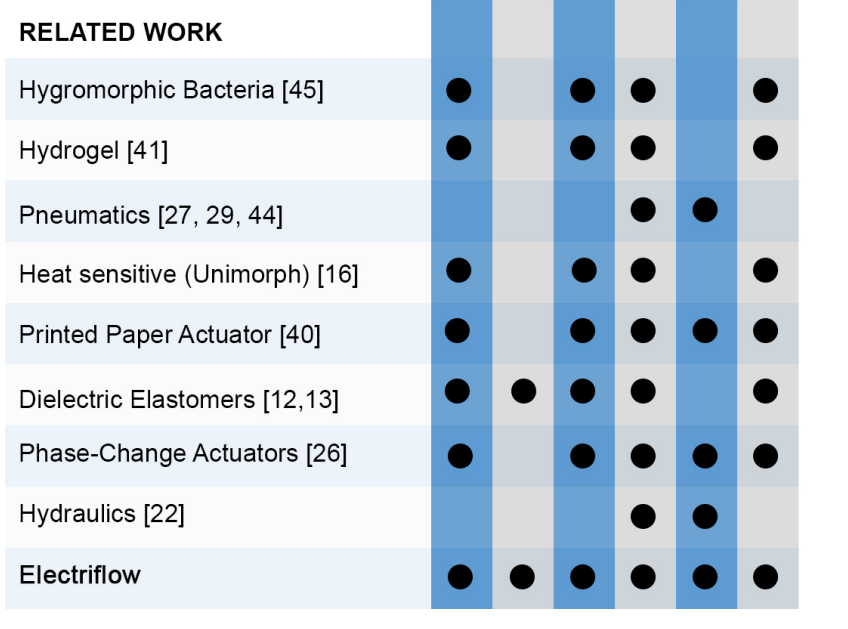

Figure 2: A comparison of various types of prototyping technologies for bio-inspired shape-changing interfaces.

Pneumatics offer an alternative method for prototyping bioinspired shape-changing interfaces. Pneumatic actuators have been used for transformable phones and lights [44], origami-inspired shape change [27, 29], haptic jackets [10], and shape-change garments [31]. Despite their popularity, pneumatic actuators require 

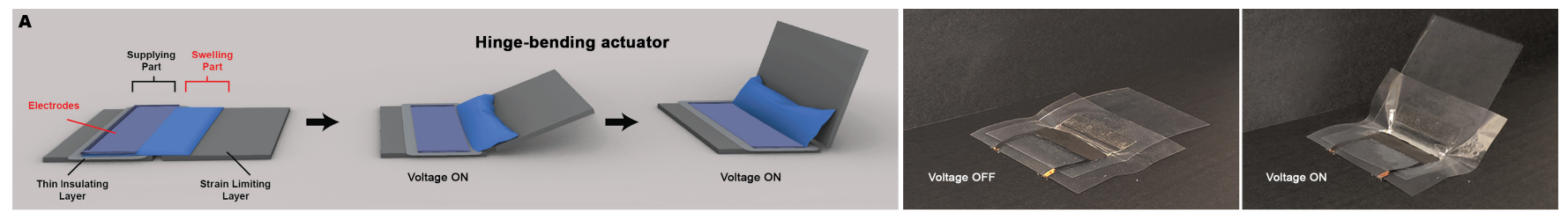

B
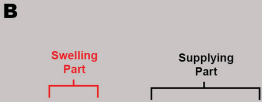

Crescent Actuator

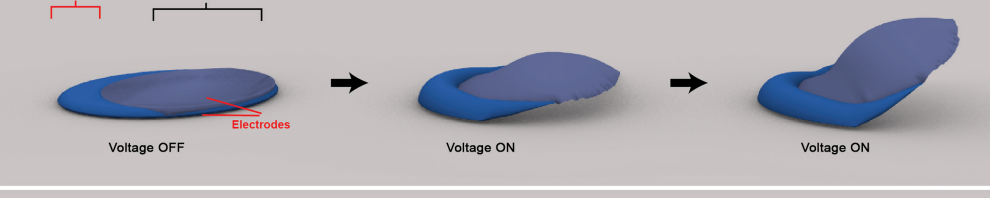

C
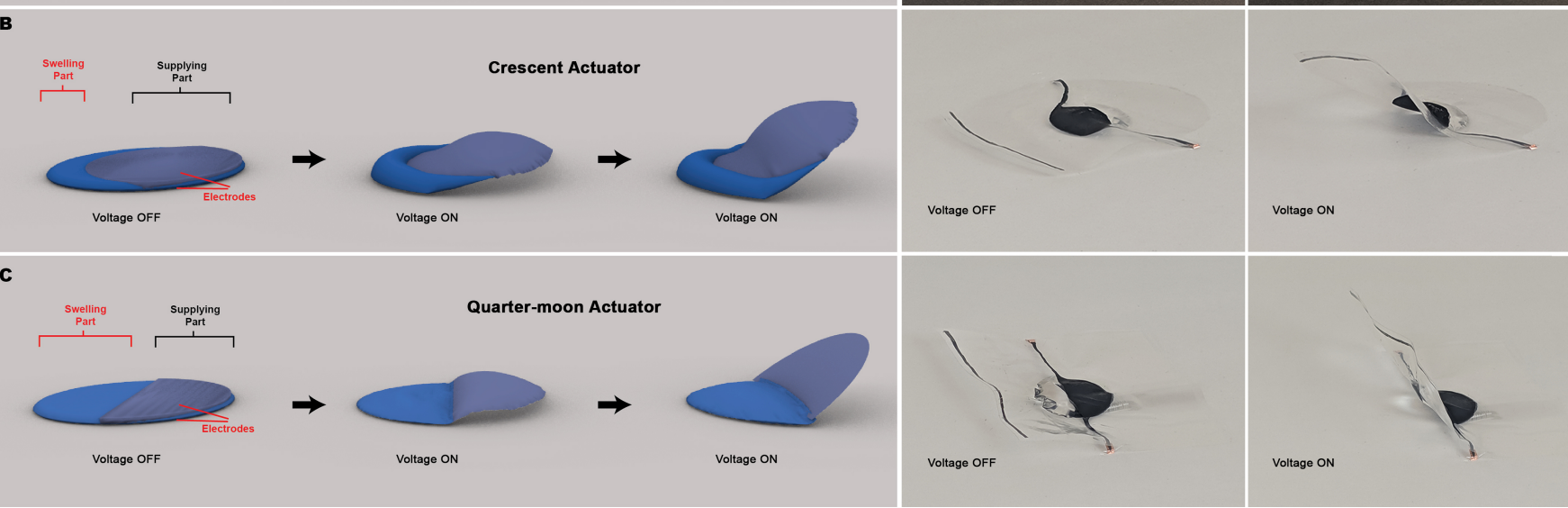

Figure 3: Three types of electrohydraulic actuators as building blocks for prototyping shape change. (A) Shows the main components of the Hinge-Bending Actuator and its actuation states along with the actuator images in OFF and ON state. (B) shows the main components of the Crescent actuator along with actuation states as voltage is applied while (C) shows the same for Quarter-moon actuator.

an external fluid source for inflation and deflation, so they must be tethered through tubing to rigid, noisy, bulky pumps and valves, limiting their speed, noise level, and portability. Pumping alternatives such as chemical reactions have been explored [42] for pneumatic inflation, but these options lead to slower response times with irreversible shape change. Similar to pneumatics, actuators which use liquids as working fluids have also been proposed like hydraulic actuators [22] or phase-change actuators [26]. However, they are also slow in response time.

A silent and rapid actuating technology, recently explored for prototyping bioinspired shape-changing interfaces are Dielectric Elastomer Actuators (DEA).

DEAs are activated by electrostatic voltage, are rapid and silent in actuation. They have been explored for applications in the domain of haptics [46], handheld shape-changing devices [35], and responsive environments [14]. However, they are limited in availability due to their high cost of the material and specific know-how of fabrication [13]. Figure 2 highlights the advantages of Electriflow over comparable prototyping technologies for bioinspired shapechanging interfaces.

\subsection{Electrohydraulic Actuators}

To address the demand for high-speed silent actuation and portability, electrohydraulic actuators have been proposed for various applications in design and engineering. These actuators exploit the advantages of conventional hydraulic systems as well as directdrive electrical actuators [15]. Traditionally, such actuators have been constructed from rigid electromechanical components [12], but recently soft versions have also been introduced [1]. While soft hydraulic actuation has been investigated for its basic working principle $[1,38]$ and for robotic applications [19, 38], its application to human-machine interfaces has been limited to haptic devices $[21,37]$. There is a need to bridge the gap between material science technology and user-centered design practices for these actuators, just as previous HCI work has done for hygromorphic bacteria $[9,45]$, or triboelectric nanogenerators $[6,7,17,47]$.

In the following section, we present the detailed design, construction, and operating principle of Electriflow for shape-changing interfaces. Electriflow is inspired by the electrical characteristics of hydraulically amplified self-healing electrostatic (HASEL) actuators, $[1,19]$ which operate by displacing fluid inside of flexible pouches, and can be fabricated through inexpensive tools and commodity materials [25]. We also present a software tool to facilitate the design and fabrication process in order to streamline the prototyping workflow for shape-changing interfaces.

\section{ELECTRIFLOW: ELECTROHYDRAULIC BUILDING BLOCKS}

Electriflow utilizes a combination of electrostatic and hydraulic phenomena to constrain the flow of a working fluid contained in flexible pouches. Based on this working mechanism, we introduce three novel bending actuators as building blocks for prototyping shape-changing interfaces: a Hinge Bending actuator, a Crescent actuator, and a Quarter Moon actuator shown in figure $3 \mathrm{~A}, 3 \mathrm{~B}$, and $3 \mathrm{C}$ respectively.

These compact, silent actuators produce immediate and rapid shape change. In this section, we first present the generalized design of an actuator using the Hinge Bending actuator as an example. 


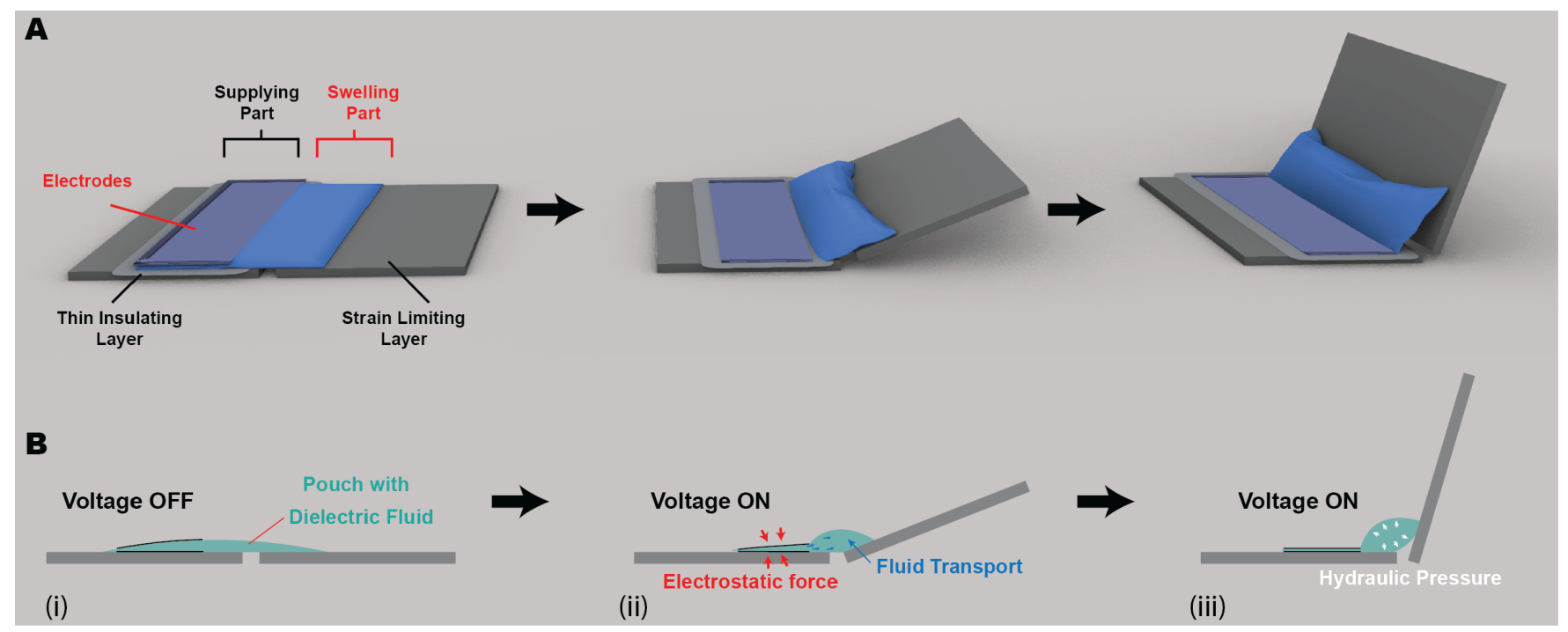

Figure 4: Working principle of electrohydraulic actuators for prototyping shape change. (A) Shows the main components of the Hinge-Bending Actuator and its actuation states. These components represent the most generic construction of Electriflow. (B) Shows the principle of electrohydraulic actuation with electrode zipping as voltage is applied.

Afterwards, we discuss the operating principle and specific actuator geometries.

\subsection{Actuator Construction}

Our general electrohydraulic actuator consists of four main parts as follows:

- a pouch that is non-stretchable and compliant (figure $4 \mathrm{~B}(\mathrm{i})$ )

- flexible electrodes covering parts of the pouch (shown in purple in figure 4A)

- a rigid strain limiting layer attached to one side of the pouch (shown in dark grey in figure $4 \mathrm{~A}$ )

- a thin insulating layer between the electrodes and the strain limiting layer (shown in light grey in figure 4A)

Note that the strain-limiting layer and the thin insulating layer may be absent in appropriately designed actuators such as the Crescent (figure 3B) and the Quarter-moon (figure 3C) and still produce bending behavior. The pouch is filled with a dielectric liquid (silicone oil in this case) and is divided into two regions: a supply region (purple in figure 4A and figure 3), and a swelling region (blue in figure $4 \mathrm{~A}$ and figure 3 ). The supply region is covered with opposing flexible electrodes on both sides. The combination of supply and swelling geometry and the 'strain limiting layer' (when present) gives rise to different shape outputs for the actuators (see figure 3).

\subsection{Operation Principle}

The operation principle is illustrated in figure $4 \mathrm{~B}$. When the electrodes on the supply region are oppositely charged by a highvoltage input ( $10 \mathrm{kV}$ in this case), the electrostatic force drives the electrodes progressively toward each other in a zipping motion (figure $4 \mathrm{~B}$ (ii)). This zipping motion displaces the dielectric fluid in the supply region into the swelling region. This process continues with increasing applied voltage until equilibrium is reached between the electrostatic forces between electrodes and restoring hydraulic pressure (see figure $4 \mathrm{~B}(\mathrm{iii})$ ). The local pressure increase causes the swelling region to inflate, and the cross-section of the pouch changes from a thin ellipse to a thick ellipse (see figure 4 $\mathrm{B}(\mathrm{iii})$ ). This shape change in the pouch results in angular deflection of the actuator. In the case of Hinge-bending actuator, it is supplemented by the rigidity of the strain-limiting layer. The Crescent and the Quarter-moon actuators produce bending behavior due to asymmetric position of supplying region and swelling region. All three kinds of actuators in ON and OFF states are illustrated in figure 3 .

\subsection{Building Blocks for Shape Change}

We introduce three novel actuators with bending behavior as shapechanging building blocks: a Hinge Bending actuator, a Crescent actuator, and a Quarter Moon actuator.

The Hinge Bending actuator, obtained by attaching a strainlimiting layer to a rectangular pouch, produces sharp bends (figure 3 and 4 ).

The Crescent actuator has a circular fluid-filled region and a non-concentric elliptical electrode, resulting in an asymmetric swelling region that drives a smooth-curve bending behavior (figure 3).

Building upon the same principles, the circular fluid-filled pouch of the Quarter Moon Actuator is bisected by the line that divides the symmetrical semicircles defining its supply and swelling regions. This actuator geometry results in a sharp bending behavior, as shown in figure 3C. 


\subsection{Technical Evaluation}

We evaluated the shape-changing behavior of our actuators for bending angle, response time and strength. The bending angle is plotted against applied voltage in figure 5). A Crescent actuator can produce an smooth organic bend with a maximal bending angle of around $60^{\circ}$ while the Quarter Moon actuator produces sharp bends with bending angle of around $40^{\circ}$.

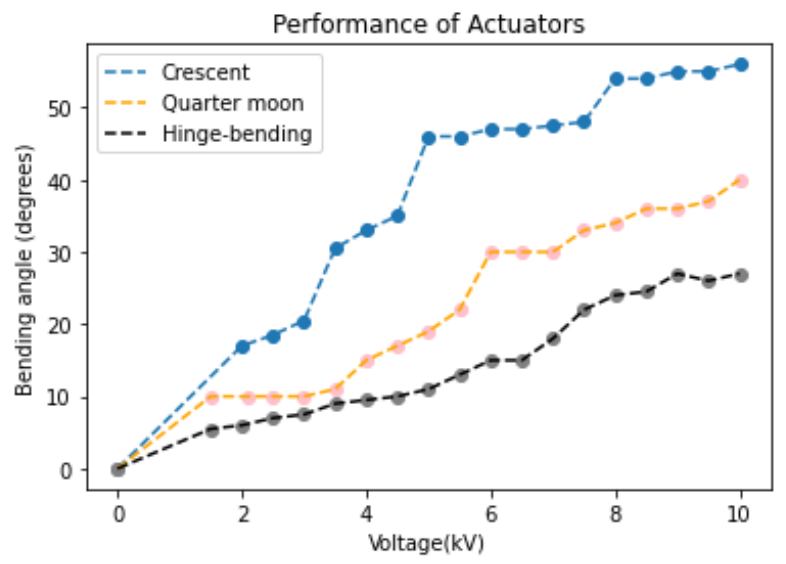

Figure 5: Dependance of bending angle on activation voltage for Crescent actuator and Quarter-moon actuator and the Hinge-bending actuator.

The actuation speed of all the building blocks was consistent with the maximum frequency of stimulation at $25 \mathrm{~Hz}$ (see video for details). For the Hinge-bending actuator the maximum weight lifted without reduction in bending angle was 13 grams (for a pouch with dimension $2 \mathrm{~cm} \mathrm{x} 4 \mathrm{~cm}$ and liquid volume of $3 \mathrm{ml})$.

\subsection{Materials}

The construction of electrohydraulic actuators is highly dependent on the materials used for their components. The outer pouch that is non-stretchable and compliant is constructed out of heat sealable PET (polyethylene terephthalate; thickness, $22 \mu \mathrm{m}$ ) sheets. The dielectric fluid used in this work was silicone oil (Super Lube, 100 $\mathrm{cSt}$ ). The flexible electrodes were painted using conductive carbon paint (Ted Pella, ELECTRODAG T-502, 30G). The strain limiting layer has two types: one constructed from transparency sheets (C-Line 60837) and the other using rigid acrylic blocks (thickness $6 \mathrm{~mm})$. The thin insulating layer between the electrodes and the strain limiting layer are constructed out of transparency sheets (C-Line 60837).

An electrostatic control system (see Fig. 8B) drives the electrohydraulic actuators. This modular power supply unit was based on the open source power supply developed by PetaPicoVoltron [36].

\section{ELECTRIFLOW FOR PROTOTYPING INTERACTIVE SYSTEMS}

Electriflow actuators act as a building blocks for prototyping shapechanging interfaces. The Hinge Bending actuator uses a strainlimiting layer to produce sharp fold-like shape change and can be attached to existing objects to actuate them (see application prototypes of actuating origami creases or the shape-changing bowl). The Crescent actuator produces smooth curve-like folds and the Quarter Moon actuator produce sharp folds without the semi-rigid 'strain-limiting layer' which makes them suitable for applications where softness of the actuator is important (see application prototypes of actuating butterfly in figure 11 and character animation in figure 12).

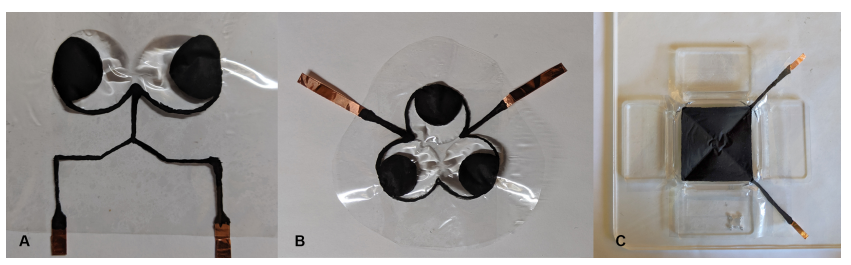

Figure 6: The Electriflow building blocks can be arranged in different configurations depending on applications. (A) Show two Crescent actuators side-bu-side facing opposite to each other; (B) or in an equilateral triangle facing outward; (C) four hinge bending actuators arranged around the edges of a square.

Electriflow building blocks can also be combined in different orientations to produce shape-changing structures that are more complex in shape geometry and output. For instance, we demonstrate the utility of the Crescent actuators as building blocks by grouping them in different configurations. These assemblies become the secondary primitives for other shape-changing objects. Figure 6A shows two Crescent actuators arranged side-by-side facing opposite to each other. This arrangement becomes the bending primitive for the constructing a butterfly (see Prototyping Application section and figure 11). Figure $6 \mathrm{~B}$ shows three Crescent actuators arranged at the corners of an equilateral triangle. This arrangement becomes the bending primitive for the flower petal (see Prototyping Application section and figure 13). Similarly a group of Hinge-bending actuators can be arranged at the sides of a square (figure 6C) to form the basis of a shape-changing bowl (figure 17).

\section{SOFTWARE TOOL}

To supplement the Electriflow building blocks, we developed a software design tool to enable designers and $\mathrm{HCI}$ practitioners to prototype their own shape-changing interfaces by visualizing the design, simulating actuation, and aiding the fabrication of specific shape-changing structures. The main motivation for the software tool is to streamline a user workflow from real-time visualization of iterative designs to the fabrication process. We built the tool in the Rhinoceros 3D environment with Grasshopper plug-in. Users can import their own pouch and electrode geometries, visualize the shape output of their actuator, and export the files that direct the fabrication process-for example, heat-sealing the outer polymeric shell, fluid volume, and electrode outlines. The tool enables the following user workflow: 

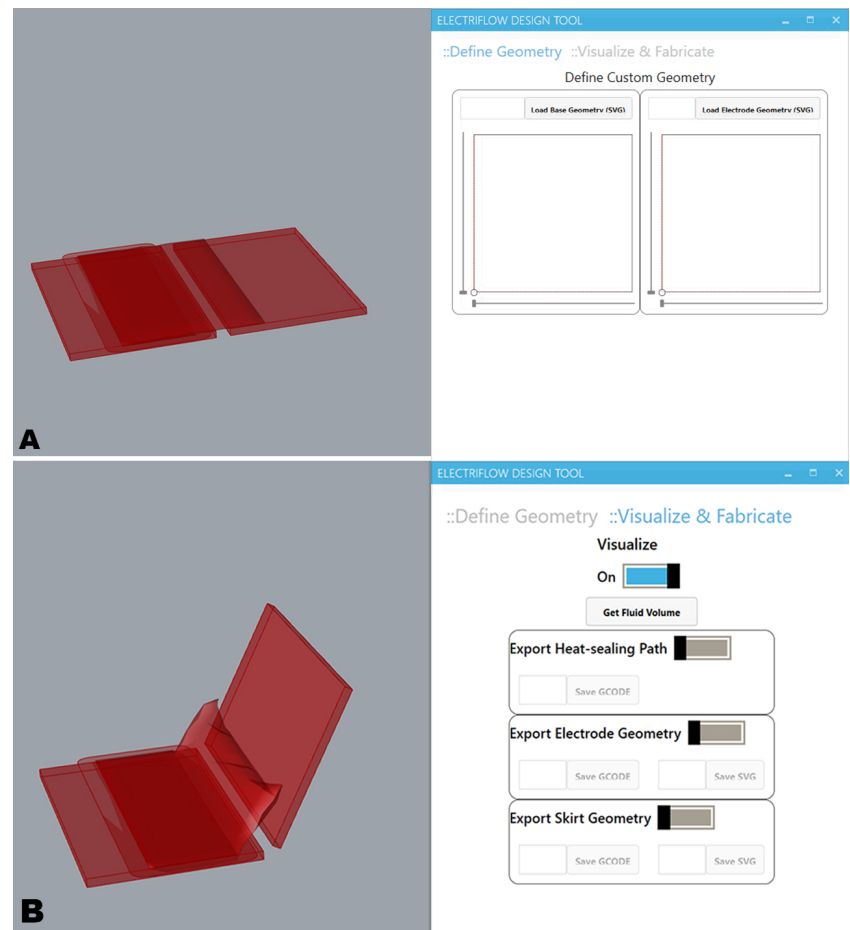

Figure 7: Software Design and Visualization tool built in Rhinoceros 3D with Grasshopper environment. (A) The user can play import custom geometry as SVG file. (B) They can also visualize the shape change and export fabrication files.

\subsection{Design}

A user begins by defining the geometry of the shape-changing actuator to be visualized and fabricated. This geometry is defined by planar curves that outline the edges of the fluid region (pouch) and the electrodes. To define these curves the user can import the base geometry of the pouch as an SVG file. The user can further define the electrode area by importing another SVG for the electrode outline. When a geometry is imported, the user-defined shape-changing object is automatically generated and displayed on the design view.

\subsection{Visualize Shape}

To display the shape output, the user remains in the Visualization window and presses the 'Visualize' button.

\subsection{Export Fabrication Files}

After the shape visualization, the user can view or export a series of files that facilitate actuator construction as described below in the Fabrication Pipeline section.

Figure 9 illustrates the step-by-step design, visualization and fabrication workflow for an example prototype involving character animation [see the Prototyping Application section below for more details]. In Figure 7 we show the relevant sections of our software tool.

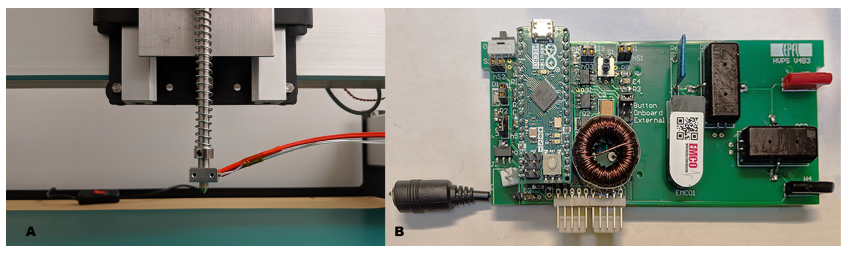

Figure 8: Electrohydraulic actuators are a hybrid of fluiddriven actuators and EAPs. They can be fabricated by (A) Custom Heat-Sealing Tool and (B) powered by a DC high voltage power supply [36]

\section{FABRICATION PIPELINE}

The current fabrication process for the electrohydraulic actuators involves automated as well as manual processes. The software tool helps in each of the following fabrication steps.

\subsection{Heat-sealing outer shell}

The software tool generates ' $g$-code' for the path defining the outer edges of the pouch to be heat sealed. This g-code file is directly accepted by a customized heat-sealing CNC.

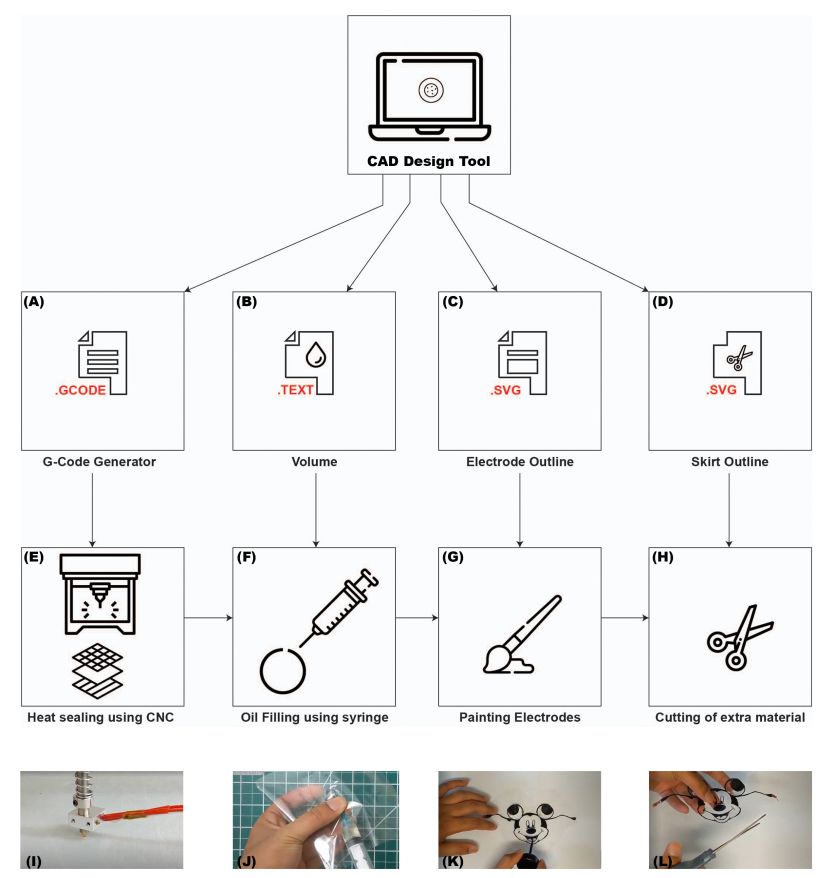

Figure 9: CAD Design Tool assisted fabrication pipeline for electrohydraulic shape-changing interfaces.

To fabricate the pouch's polymeric shell, two sheets of thin-film polymer are placed on top of a thick soldering mat and beneath a thin teflon sheet. The polymer films are bonded together by the heat sealing head of the $\mathrm{CNC}$ machine as it traces the edges of the pouch. The soldering mat facilitates smooth sealing by cushioning 


\begin{tabular}{|c|c|c|c|}
\hline APPLICATION PROTOTYPE & DESIGN RATIONALE & ACTUATOR USED & ACTUATOR PROPERTY \\
\hline Butterfly (Tangible Animation) & Need large bending angle with organic shapes & Crescent Actuator & $\begin{array}{l}\text { Produces large bending angle with organic shapes; } \\
\text { Can't be attached to existing objects }\end{array}$ \\
\hline Flower Petal (Tangible Animation) & Need Organic Shapes with smooth bends & Crescent Actuator & $\begin{array}{l}\text { Produces large bending angle with organic shapes; } \\
\text { Can't be attached to existing objects }\end{array}$ \\
\hline Character Animation & Need Organic Shapes & Crescent Actuator & $\begin{array}{l}\text { Produces large bending angle with organic shapes; } \\
\text { Can't be attached to existing objects }\end{array}$ \\
\hline Shape-changing Phone & Need Sharp Bending Angle and compact design & Quarter-Moon Actuator & Produces sharp bends; Can't be attached to existing objects \\
\hline Shape-changing Bowl & $\begin{array}{l}\text { Need an actuator design which could be attached } \\
\text { to different kind of hinges to actuate them }\end{array}$ & Hinge-bending Actuator & Produces sharp bends; Can be attached to existing objects \\
\hline
\end{tabular}

Figure 10: Summary of applications prototyped, emphasizing on their design rationale, type of actuator used and their properties. All application prototypes are described in detail in the Prototyping Applications Section.

the downward pressure applied by the heat sealing tip, while the teflon sheet prevents damage to the pouch by reducing friction and avoiding direct contact between the hot tip and the thin plastic sheets. The heat-sealing head is a custom modified extrusion head of a 3D printer. The heat sealed curve is not closed, leaving open a small input port to fill with working fluid.

\subsection{Filling liquid dielectric}

The software tool automatically calculates the volume of the working fluid to be filled inside the actuator. This liquid (in our case silicone oil) is filled manually in the heat-sealed pouch by syringe. Care must be taken to ensure that no air bubbles form inside the pouch, since the compressibility of air can lead to unpredictable volume change. After liquid filling, the input port is manually heat sealed with a soldering iron or an impulse sealer.

\subsection{Adding Electrodes}

The software tool guides the shape of the electrode to be added by providing the geometry. A user can export SVG or gcode files to fabricate an electrode stencil with manual or digital tools, respectively. The flexible electrodes can be painted with a commercially available conductive ink or other forms of flexible electrodes. Since the circuit is capacitive for electrohydraulic actuators, the electrostatic activation has low sensitivity to electrode resistance. We typically apply hand-painted conductive carbon paint for our leads and electrodes.

\subsection{Cutting and leaving "skirt"}

Finally, the excess plastic is cut from the outer shell, leaving an insulating "skirt" around the edges that ensures a minimum throughair distance between electrodes to prevent arcing. The software can automatically compute the skirt dimensions based on the dielectric breakdown strength of air $(3 \mathrm{kV} / \mathrm{mm})$, and allow the user to export the skirt geometry as SVG or g-code files.

\section{PROTOTYPING APPLICATIONS}

The high speed, compact size, and silent actuation abilities of Electriflow combined with the simplified fabrication pipeline enabled by the software tool makes it a powerful technology for prototyping shape-changing interfaces. To highlight these features, we propose a number of application prototypes like tangible animation (a soft butterfly robot, physical animation of characters embedded in a book), mock-up of a shape-changing phone, and a shape-changing bowl. We present their summary along with their design rationale in figure 10 .

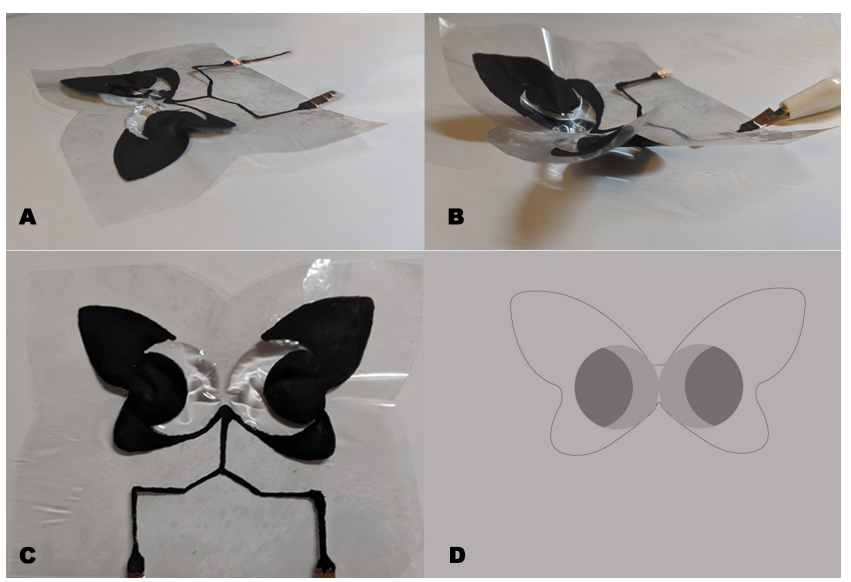

Figure 11: An actuating butterfly for tangible animation. (A) State when the voltage is OFF, (B) state when the voltage is $\mathrm{ON},(\mathrm{C})$ the top view and (D) the actuator layout consisting of two crescent actuators facing opposite to each other.

\subsection{Tangible Animation}

7.1.1 Soft Biomimmetic Butterfly Robot. Butterflies are fascinating organisms given their ability to rapidly actuate their wings. The typical butterfly wings can actuate at a frequency of around 10 $\mathrm{Hz}$ but for certain species of moths, it can go up to $26.1 \mathrm{~Hz}$ [39]. Electroflow actuators can be a great prototyping technology for the design of a bioinspired butterfly soft robot given their compact design and high frequency of actuation. Here, we show an example prototype of a butterfly, composed of two Crescent actuators embedded in the wings that can actuate up to a frequency of 25 $\mathrm{Hz}$ that is comparable to the ones found in nature. The electrode geometry has been modified according to the shape of butterfly wings. At an activation voltage of $10 \mathrm{kV}$, the fluid contained in the wings is displaced toward the body of the butterfly, resulting in flapping motion (see Fig. 11). 


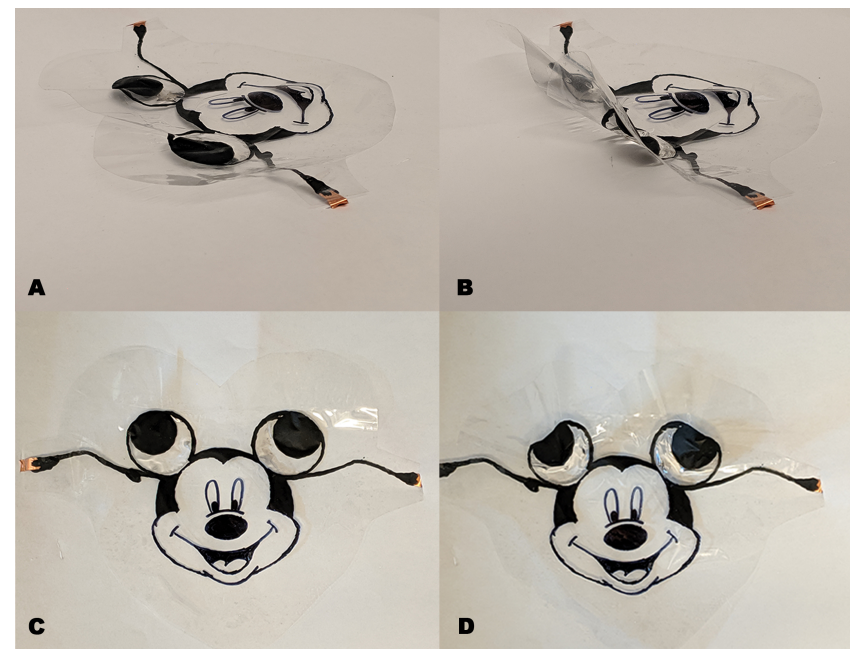

Figure 12: Embedded Crescent actuator on thin film sheets for character animation with Voltage $\operatorname{OFF}(A, C)$; and Voltage ON (B,D). Each of the ears has an actuator underneath.

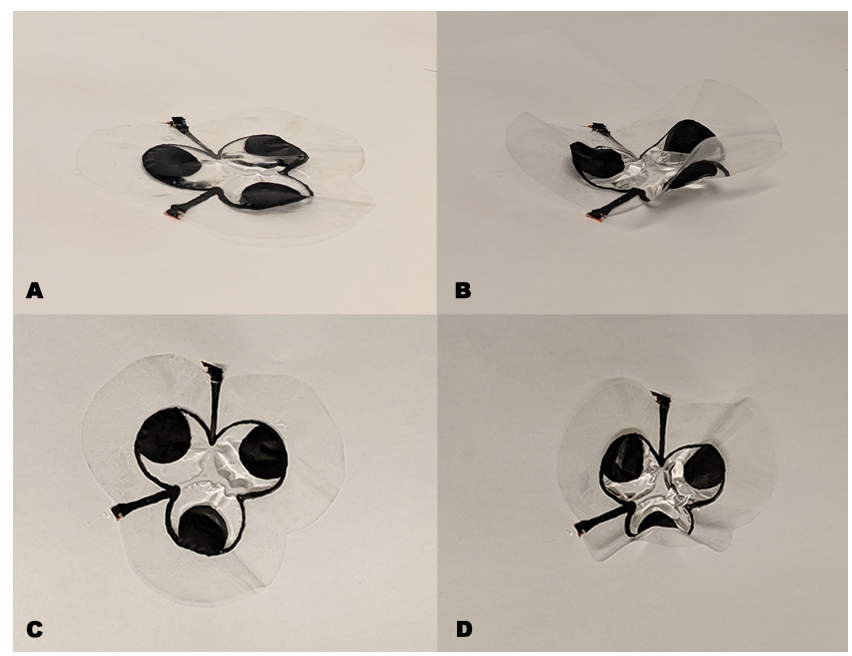

Figure 13: Crescent actuator used as a Curling Flower Petal with Voltage OFF (A,C); and Voltage ON (B,D).

7.1.2 Physical character animation. The compact design of Crescent actuators ensures they can be embedded on thin-film sheets for character animation. For instance they can actuate physical characters as shown in figure 12. Here, each of the ears has an actuator underneath.

7.1.3 Curling Flower Petal. Crescent Actuators can be combined in wide variety of numbers and configurations, depending upon the need of prototyping. For example, a group of crescent actuators forms the basis of a flower that blooms (see figure 13). Each Crescent actuator acts as a petal of the flower. A collection of three Crescent actuators arranged in a triangular lattice (at the vertices of an equilateral triangle) become the fundamental unit of the flower. The flower blooms when the voltage is applied as shown in Figure 13.

The compact design of the actuators and the power supply enables embedding these soft objects into a variety of form-factors. We also integrated the butterfly and the flower petal into a prototype for a book which could provide the tangible animation (see figure 14). The power supply is also embedded inside the book.

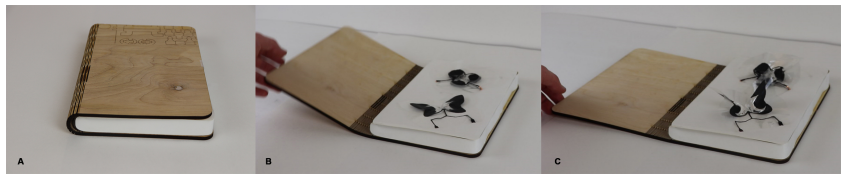

Figure 14: Tangible animation integrated in the prototype of a book.

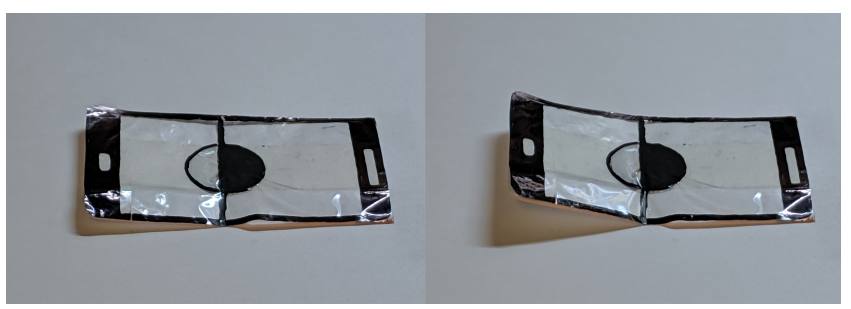

Figure 15: Example of a shape-changing display mockup based on the Quarter Moon Actuator

\subsection{Shape-Changing Display}

Flexible displays enable new opportunities for interaction with mobile devices [20]. Adding shape-changing abilities adds a new output dimension for haptic notifications and information display [30, 35, 44]. To explore a thin form factor flexible display, we prototyped a shape-changing mockup with an embedded bending quarter moon actuator (see figure 15). The quarter moon actuator ensures a sharp bend for the display, without needing an strain limiting layer. We hope in the future, Electriflow may act as the basis for actuating such a flexible display. We imagine a scenario when a phone with such a display is placed on the table and a notification arrives, the phone bends upwards to silently alert the user. This bending behaviour can also pulse at different frequencies to convey urgency.

\subsection{Actuating Origami}

Our hinge bending electrohydraulic actuators have been designed to turn the creases of origami folds into active hinges. Some example prototypes based on this approach are shown in Fig. 16. A single sheet is folded into an origami crane, and the actuators are attached to the creases of the wings to move them. Likewise, a sheet folded into a box is augmented with actuators that open and close its lid. In both cases, the actuator transforms a static object into a kinetic sculpture. Numerous further origami designs exist that can 


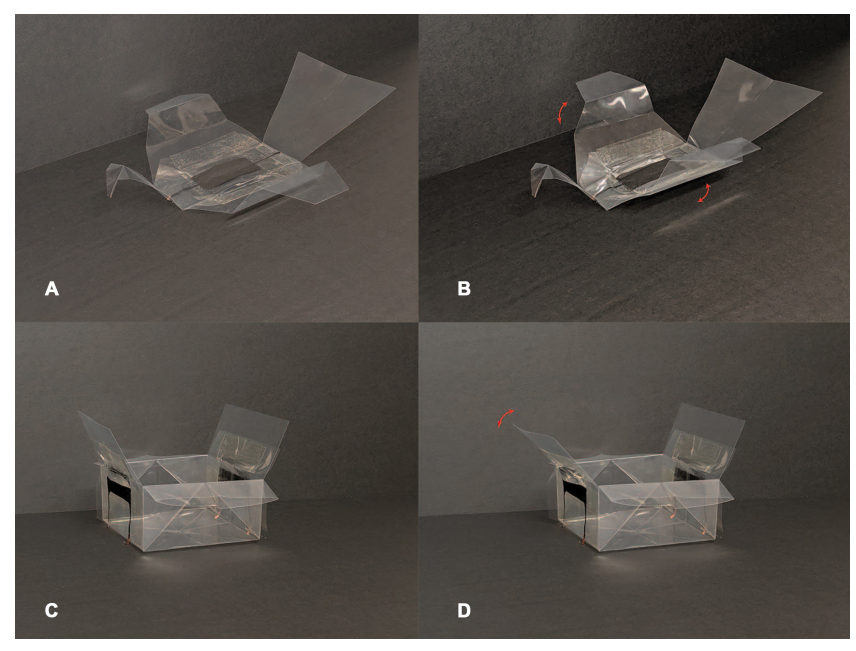

Figure 16: Using electrohydraulic actuators to actuate origami. (A) An origami crane with Voltage OFF, (B) with Voltage ON; (C) a box flap with Voltage OFF, (D) with Voltage $\mathrm{ON}$.

be augmented through this technique in a calm, silent and unobtrusive matter. We envision applications for such kinetic origami in areas such as art, information physicalization, and tangible user interfaces.

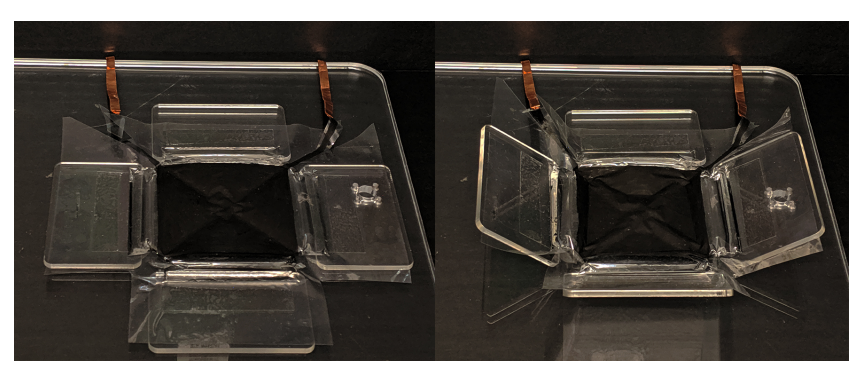

Figure 17: Example of shape-changing bowl

\subsection{Shape-Changing Bowl}

Hinge bending actuator requires a strain limiting layer to produce sharp bends. Consequently, it can be attached to existing objects and rigid hinges to actuate them. A group of such actuators can be arranged to form a prototype of a shape-changing fruit bowl that transforms between a deep and shallow shape on demand (Fig. 17). The bowl is made of transparent acrylic panels that are connected to each other through electrohydraulic hinges. When small fruits are placed inside the bowl, the actuators lift the acrylic side panels to form a deeper bowl. When a large fruit is placed in the bowl, it transforms to a large, shallow shape by lowering the side panels.

\section{LIMITATIONS AND FUTURE WORK}

Electriflow presents a compact, fast and silent method of actuating everyday objects and prototyping shape-changing interfaces. While it augments the design of interactive systems, in course of prototyping applications we also came across a number of limitations. We plan to address them in future work.

\subsection{Voltage of Actuation}

At present the stimulation of our electrohydraulic actuators still occurs at high voltages $(6-10 \mathrm{kV})$ which offers many challenges for day-to-day use and safety. Though the control electronics ensures these actuators are non-lethal if touched, there is still plenty of room for improvement such that the users are able to touch them. An active area of research in this direction is developing actuators which operate at lower voltages which can be achieved with a different combination of pouch material and dielectric liquid [34]. These improved actuators would also simplify the control electronics as switching high voltage circuits require special components.

Another way to address this challenge is by providing a suitable layer, as proposed in related work on haptics [21,37]. An important point to note here is that, the activation voltage in such systems is significantly lower $(1-2 \mathrm{kV})$, making insulation easy in comparison to the activation voltage of actuators.

\subsection{Lack of Interactivity}

In this work, we primarily focused on prototyping the output of shape-changing interfaces and less on sensing user input. Adding input sensing modalities such as embedded touch sensing would greatly increase the interactive capabilities. For future work, incorporating the sensing methods proposed by $[23,44]$ (e.g., acoustic, capacitive, and pressure sensing) should be explored to improve the interactivity of the current prototype. The inherent design of electrohydraulic actuators with capacitive electrodes offer possibilities for self sensing [23]. However, we have not yet explored the sensing capabilities in electrohydraulic actuators due to the high voltage, which requires custom sensing electronics. We are also interested in using a dynamic appearance (e.g., using colored working liquid in the electrohydraulic actuators) to provide different affordances.

\subsection{User Evaluation}

All of our application prototypes has been created by ourselves and we have not formally evaluated them with a user study. We also understand the need for the user evaluation of the software tool. As a next step, we plan a workshop with HCI researchers and designers to explore and prototype, similar to related work [13, 14]. We expect a formal evaluation to provide insights into how these building blocks support design ideation.

\subsection{Extension of Shape-changing Behaviors}

Our software visualization tool enables HCI researchers to explore further types of shape-changing behaviors. A broader and systematic exploration of shape-changing building blocks could increase use cases in the design of future user interfaces. For example, topological change, including creating holes on surfaces, can give interesting affordances for interaction. Locomotion as a bio-inspired 
mechanism can be achieved with programmable constraints in the material structure.

\subsection{Programmable Shape-changing Interfaces}

The current fabrication and insulation process allows the interfaces to morph between two shapes. It is still desired that the interface can have multiple shape-changing states that can be dynamically controlled in real-time. One way to achieve it could be by introducing other active stimuli. Multiple actuating states has the potential to become one of the major advantages of the of electrohydraulic actuators. The electrohydraulic mode of activation opens up scope for multiple types of stimuli (different signals and voltages) to achieve active, fully programmable shape change. Another opportunity is to increase the number and resolution of the actuators. A bottleneck for high-resolution actuator configurations is a flexible insulation of electrodes as they suffer from arcing at high voltages when they are placed close to each other. We hope that with a better conformal insulating layer we will move a step closer towards programmable, dynamically controllable, shape-changing interfaces.

\section{CONCLUSION}

We have presented Electriflow, electrohydraulic actuators as building blocks for prototyping shape-changing user interfaces. Based on our novel shape-changing actuators, we demonstrate how these building blocks can enable new types of human computer interfaces. We also proposed a software tool to aid the design, visualization, and fabrication of shape-changing objects. To demonstrate the application space enabled by electrohydraulic actuators, we presented prototypes for tangible animation integrated in a book, shape-changing mobile devices and bowl, and origami actuation. We envision these electrohydraulic building blocks can be leveraged by designers and other HCI researchers and open new possibilities for prototyping shape-changing interfaces.

\section{ACKNOWLEDGMENTS}

This work was supported by funding from the College of Engineering and Applied Science at the University of Colorado Boulder We thank Nivedita Arora for valuable suggestions in the draft and proof-reading.

\section{REFERENCES}

[1] E Acome, SK Mitchell, TG Morrissey, MB Emmett, C Benjamin, M King, M Radakovitz, and C Keplinger. 2018. Hydraulically amplified self-healing electrostatic actuators with muscle-like performance. Science 359, 6371 (2018), 61-65.

[2] Jason Alexander, Anne Roudaut, Jürgen Steimle, Kasper Hornbæk, Miguel Bruns Alonso, Sean Follmer, and Timothy Merritt. 2018. Grand challenges in shape-changing interface research. In Proceedings of the 2018 CHI conference on human factors in computing systems. 1-14.

[3] Justine J Allen, George RR Bell, Alan M Kuzirian, and Roger T Hanlon. 2013. Cuttlefish skin papilla morphology suggests a muscular hydrostatic function for rapid changeability. Journal of morphology 274, 6 (2013), 645-656.

[4] Justine J Allen, George RR Bell, Alan M Kuzirian, Sachin S Velankar, and Roger T Hanlon. 2014. Comparative morphology of changeable skin papillae in octopus and cuttlefish. Journal of morphology 275, 4 (2014), 371-390.

[5] Byoungkwon An, Ye Tao, Jianzhe Gu, Tingyu Cheng, Xiang'Anthony' Chen, Xiaoxiao Zhang, Wei Zhao, Youngwook Do, Shigeo Takahashi, Hsiang-Yun Wu, et al. 2018. Thermorph: Democratizing 4D printing of self-folding materials and interfaces. In Proceedings of the 2018 CHI conference on human factors in computing systems. 1-12.

[6] Nivedita Arora, Thad Starner, and Gregory D Abowd. 2020. SATURN: An introduction to the Internet of Materials. Commun. ACM 63, 12 (2020), 92-99.
[7] Nivedita Arora, Steven L Zhang, Fereshteh Shahmiri, Diego Osorio, Yi-Cheng Wang, Mohit Gupta, Zhengjun Wang, Thad Starner, Zhong Lin Wang, and Gregory D Abowd. 2018. SATURN: A thin and flexible self-powered microphone leveraging triboelectric nanogenerator. Proceedings of the ACM on Interactive, Mobile, Wearable and Ubiquitous Technologies 2, 2 (2018), 1-28.

[8] Elizabeth L Brainerd. 1994. Pufferfish inflation: functional morphology of postcranial structures in Diodon holocanthus (Tetraodontiformes). fournal of morphology 220, 3 (1994), 243-261.

[9] Xi Chen, Lakshminarayanan Mahadevan, Adam Driks, and Ozgur Sahin. 2014. Bacillus spores as building blocks for stimuli-responsive materials and nanogenerators. Nature nanotechnology 9, 2 (2014), 137-141.

[10] Alexandra Delazio, Ken Nakagaki, Roberta L Klatzky, Scott E Hudson, Jill Fain Lehman, and Alanson P Sample. 2018. Force jacket: Pneumatically-actuated jacket for embodied haptic experiences. In Proceedings of the 2018 CHI Conference on Human Factors in Computing Systems. 1-12.

[11] Yoël Forterre, Jan M Skotheim, Jacques Dumais, and Lakshminarayanan Mahadevan. 2005. How the Venus flytrap snaps. Nature 433, 7024 (2005), 421-425.

[12] Michel Franchet, Daniel Kettler, and Pascal Lejeune. 2004. Electrohydraulic actuator. US Patent 6,796,120.

[13] Karmen Franinović and Luke Franzke. 2019. Shape Changing Surfaces and Structures: Design Tools and Methods for Electroactive Polymers. In Proceedings of the 2019 CHI Conference on Human Factors in Computing Systems (Glasgow, Scotland Uk) (CHI '19). Association for Computing Machinery, New York, NY, USA, 1-12. https://doi.org/10.1145/3290605.3300355

[14] Karmen Franinović, Luke Franzke, Florian Wille, and Andrés Villa Torres. 2019. Interacting with Electroactive Polymers in Responsive Environments. In Proceedings of the Thirteenth International Conference on Tangible, Embedded, and Embodied Interaction (Tempe, Arizona, USA) (TEI '19). Association for Computing Machinery, New York, NY, USA, 505-512. https://doi.org/10.1145/3294109.3301268

[15] Saeid Habibi and Andrew Goldenberg. 1999. Design of a new high performance electrohydraulic actuator. In 1999 IEEE/ASME International Conference on Advanced Intelligent Mechatronics (Cat. No. 99TH8399). IEEE, 227-232.

[16] Felix Heibeck, Basheer Tome, Clark Della Silva, and Hiroshi Ishii. 2015. uniMorph: Fabricating thin film composites for shape-changing interfaces. In Proceedings of the 28th Annual ACM Symposium on User Interface Software \& Technology. 233-242.

[17] Mustafa Emre Karagozler, Ivan Poupyrev, Gary K Fedder, and Yuri Suzuki. 2013. Paper generators: harvesting energy from touching, rubbing and sliding. In Proceedings of the 26th annual ACM symposium on User interface software and technology. 23-30.

[18] Alan R Kay. 2017. How cells can control their size by pumping ions. Frontiers in cell and developmental biology 5 (2017), 41.

[19] Nicholas Kellaris, Vidyacharan Gopaluni Venkata, Garrett M Smith, Shane K Mitchell, and Christoph Keplinger. 2018. Peano-HASEL actuators: Musclemimetic, electrohydraulic transducers that linearly contract on activation. Science Robotics 3, 14 (2018).

[20] Byron Lahey, Audrey Girouard, Winslow Burleson, and Roel Vertegaal. 2011. PaperPhone: understanding the use of bend gestures in mobile devices with flexible electronic paper displays. In Proceedings of the SIGCHI Conference on Human Factors in Computing Systems. 1303-1312.

[21] Edouard Leroy, Ronan Hinchet, and Herbert Shea. 2020. Multimode Hydraulically Amplified Electrostatic Actuators for Wearable Haptics. Advanced Materials 32, 36 (2020), 2002564.

[22] Qiuyu Lu, Jifei Ou, João Wilbert, André Haben, Haipeng Mi, and Hiroshi Ishii. 2019. milliMorph-Fluid-Driven Thin Film Shape-Change Materials for Interaction Design. In Proceedings of the 32nd Annual ACM Symposium on User Interface Software and Technology. 663-672.

[23] Khoi Ly, Nicholas Kellaris, Dade McMorris, Brian K Johnson, Eric Acome, Vani Sundaram, Mantas Naris, J Sean Humbert, Mark E Rentschler, Christoph Keplinger, et al. 2020. Miniaturized circuitry for capacitive self-sensing and closed-loop control of soft electrostatic transducers. Soft Robotics (2020).

[24] Frederic Martini et al. 2006. Anatomy and Physiology'2007 Ed. Rex Bookstore, Inc.

[25] Shane K Mitchell, Xingrui Wang, Eric Acome, Trent Martin, Khoi Ly, Nicholas Kellaris, Vidyacharan Gopaluni Venkata, and Christoph Keplinger. 2019. An Easy-to-Implement Toolkit to Create Versatile and High-Performance HASEL Actuators for Untethered Soft Robots. Advanced Science 6, 14 (2019), 1900178.

[26] Kenichi Nakahara, Koya Narumi, Ryuma Niiyama, and Yoshihiro Kawahara. 2017. Electric phase-change actuator with inkjet printed flexible circuit for printable and integrated robot prototyping. In 2017 IEEE International Conference on Robotics and Automation (ICRA). IEEE, 1856-1863.

[27] Ryuma Niiyama, Xu Sun, Lining Yao, Hiroshi Ishii, Daniela Rus, and Sangbae Kim. 2015. Sticky actuator: Free-form planar actuators for animated objects. In Proceedings of the Ninth International Conference on Tangible, Embedded, and Embodied Interaction. 77-84.

[28] Kate Oliver, Annela Seddon, and Richard S Trask. 2016. Morphing in nature and beyond: a review of natural and synthetic shape-changing materials and mechanisms. Journal of Materials Science 51, 24 (2016), 10663-10689. 
[29] Jifei Ou, Mélina Skouras, Nikolaos Vlavianos, Felix Heibeck, Chin-Yi Cheng, Jannik Peters, and Hiroshi Ishii. 2016. aeroMorph-heat-sealing inflatable shapechange materials for interaction design. In Proceedings of the 29th Annual Symposium on User Interface Software and Technology. 121-132.

[30] Esben W. Pedersen, Sriram Subramanian, and Kasper Hornbæk. 2014. Is My Phone Alive? A Large-Scale Study of Shape Change in Handheld Devices Using Videos. In Proceedings of CHI 2014 (Toronto, Ontario, Canada). Association for Computing Machinery, New York, NY, USA, 2579-2588.

[31] Laura Perovich, Philippa Mothersill, and Jennifer Broutin Farah. 2014. Awakened Apparel: Embedded Soft Actuators for Expressive Fashion and Functional Garments. In Proceedings of the 8th International Conference on Tangible, Embedded and Embodied Interaction (Munich, Germany) (TEI '14). Association for Computing Machinery, New York, NY, USA, 77-80. https://doi.org/10.1145/2540930. 2540958

[32] Isabel P. S. Oamar, Rainer Groh, David Holman, and Anne Roudaut. 2018. HCI Meets Material Science: A Literature Review of Morphing Materials for the Design of Shape-Changing Interfaces. In Proceedings of the 2018 CHI Conference on Human Factors in Computing Systems (Montreal QC, Canada) (CHI '18). Association for Computing Machinery, New York, NY, USA, 1-23. https://doi.org/10.1145/ 3173574.3173948

[33] Majken K. Rasmussen, Esben W. Pedersen, Marianne G. Petersen, and Kasper Hornbæk. 2012. Shape-Changing Interfaces: A Review of the Design Space and Open Research Questions. In Proceedings of the SIGCHI Conference on Human Factors in Computing Systems (Austin, Texas, USA) (CHI '12). Association for Computing Machinery, New York, NY, USA, 735-744. https://doi.org/10.1145/ 2207676.2207781

[34] Philipp Rothemund, Nicholas Kellaris, Shane K Mitchell, Eric Acome, and Christoph Keplinger. 2020. HASEL Artificial Muscles for a New Generation of Lifelike Robots-Recent Progress and Future Opportunities. Advanced Materials (2020), 2003375.

[35] Anne Roudaut, Abhijit Karnik, Markus Löchtefeld, and Sriram Subramanian 2013. Morphees: toward high" shape resolution" in self-actuated flexible mobile devices. In Proceedings of the SIGCHI Conference on Human Factors in Computing Systems. 593-602.

[36] Samuel Schlatter, Patrin Illenberger, and Samuel Rosset. 2018. Peta-pico-Voltron An open-source high voltage power supply. HardwareX 4 (2018), e00039.

[37] Yitian Shao, Siyuan Ma, Sang Ho Yoon, Yon Visell, and James Holbery. 2020. Surfaceflow: Large area haptic display via compliant liquid dielectric actuators. In 2020 IEEE Haptics Symposium (HAPTICS). IEEE, 815-820.

[38] Majid Taghavi, Tim Helps, and Jonathan Rossiter. 2018. Electro-ribbon actuators and electro-origami robots. Science Robotics 3, 25 (2018).

[39] Coen Van Den Berg and Charles P Ellington. 1997. The three-dimensional leading-edge vortex of a 'hovering'model hawkmoth. Philosophical Transactions of the Royal Society of London. Series B: Biological Sciences 352, 1351 (1997), 329340 .

[40] Guanyun Wang, Tingyu Cheng, Youngwook Do, Humphrey Yang, Ye Tao, Jianzhe Gu, Byoungkwon An, and Lining Yao. 2018. Printed paper actuator: A lowcost reversible actuation and sensing method for shape changing interfaces. In Proceedings of the 2018 CHI Conference on Human Factors in Computing Systems. $1-12$.

[41] Wen Wang, Lining Yao, Teng Zhang, Chin-Yi Cheng, Daniel Levine, and Hirosh Ishii. 2017. Transformative appetite: shape-changing food transforms from $2 \mathrm{D}$ to $3 \mathrm{D}$ by water interaction through cooking. In Proceedings of the 2017 CHI conference on human factors in computing systems. 6123-6132.

[42] Penelope Webb, Valentina Sumini, Amos Golan, and Hiroshi Ishii. 2019. AutoInflatables: Chemical Inflation for Pop-Up Fabrication. In Extended Abstracts of the 2019 CHI Conference on Human Factors in Computing Systems. 1-6.

[43] Stephen E Williams and Alan B Bennett. 1982. Leaf closure in the Venus flytrap: an acid growth response. Science 218, 4577 (1982), 1120-1122.

[44] Lining Yao, Ryuma Niiyama, Jifei Ou, Sean Follmer, Clark Della Silva, and Hiroshi Ishii. 2013. PneUI: Pneumatically Actuated Soft Composite Materials for Shape Changing Interfaces. In Proceedings of the 26th Annual ACM Symposium on User Interface Software and Technology (St. Andrews, Scotland, United Kingdom) (UIST '13). Association for Computing Machinery, New York, NY, USA, 13-22. https://doi.org/10.1145/2501988.2502037

[45] Lining Yao, Jifei Ou, Chin-Yi Cheng, Helene Steiner, Wen Wang, Guanyun Wang and Hiroshi Ishii. 2015. BioLogic: natto cells as nanoactuators for shape changing interfaces. In Proceedings of the 33rd Annual ACM Conference on Human Factors in Computing Systems. 1-10.

[46] Sang Ho Yoon, Siyuan Ma, Woo Suk Lee, Shantanu Thakurdesai, Di Sun, Flávio P. Ribeiro, and James D. Holbery. 2019. HapSense: A Soft Haptic I/O Device with Uninterrupted Dual Functionalities of Force Sensing and Vibrotactile Actuation. In Proceedings of the 32nd Annual ACM Symposium on User Interface Software and Technology (New Orleans, LA, USA) (UIST '19). Association for Computing Machinery, New York, NY, USA, 949-961. https://doi.org/10.1145/3332165.3347888

[47] Junwen Zhong, Oize Zhong, Fengru Fan, Yan Zhang, Sihong Wang, Bin Hu, Zhong Lin Wang, and Jun Zhou. 2013. Finger typing driven triboelectric nanogenerator and its use for instantaneously lighting up LEDs. Nano Energy 2, 4
(2013), 491-497. 